inflammation," says Jake Glanville, CEO of Centivax, which has an anti-SARS-CoV-2 $\mathrm{mAb}$ in preclinical development. For a newly infected patient, that may be okay, he says. "You can put up with a little inflammation in exchange for better viral clearance. But that's a terrible idea to give to someone who has their lungs totally colonized." Centivax therefore knocked out effector function in its lead antibody. Lilly's second antibody, etesevimab, does this too, as do Sorrento Therapeutics' antibodies.

Another safety concern is antibody-dependent enhancement (ADE), whereby the antibody potentiates virus uptake by a macrophage, enabling entry and replication, increasing viral load and worsening disease. But evidence is mounting that the ADE risk may be small. "SARS-CoV-2 doesn't naturally target these cells [macrophages]," says University of North Carolina molecular virologist Tim Sheahan. "It's not known technically if these cells are even able to support the complete life cycle of virus replication." Hundreds of thousands of COVID-19 patients have received convalescent plasma therapy containing a wide variety of antibodies without ADE. Enhanced disease has also not been reported in human COVID-19 vaccine trials.

Nevertheless, an antibody with intact effector function could lead to an exaggerated immune response. This is a real threat to patients with advanced COVID-19. "In the very severe patients hospitalized on respirators, it probably is a concern," says Vir Biotechnology CEO George Scangos. "In newly hospitalized patients, I think the jury is still out."

To treat non-intubated patients more effectively, Vir selects its antibodies to recruit immune effector cells and mobilize them against the infection. "In all our preclinical models, effector function matters," Scangos says. Neutralizing antibodies fight infection by blocking viral entry into cells. But if these antibodies also mobilize effector cells - triggering antibody-dependent cellular cytotoxicity (by NK cells) and antibody-dependent cellular phagocytosis (by macrophages) - they also indirectly kill cells already infected by the virus. "Two ways to eliminate the infection," says Scangos. Vir expects to report phase 3 data for its lead fully human mAb, VIR7831 , by the end of January.

Vir is engineering additional effector function into its next antibody, making three Fc-region amino acid changes first described by Rockefeller University immunologist Jeff Ravetch. These mutations tighten binding to stimulatory $\mathrm{Fc}$ receptors on immune cells while reducing binding to inhibitory receptors. "The result is a dramatic increase in potency, in the short term," says Scangos. In animal models, Vir found that the engineered antibody boosts not just NK cell and macrophage cell killing, but also the $\mathrm{T}$-cell response, because the antibody Fc engages $\mathrm{Fc}$ receptors on antigen-presenting dendritic cells, says Scangos. A clinical trial is planned for early 2021.

But there's limited published evidence that anti-COVID-19 antibodies require effector function to be fully protective. A mAb therapy might not need to kill infected cells with effectors: just blocking viral entry might be enough. On the other hand, mopping up infected cells could prove necessary because not all antibodies that strongly neutralize viruses in culture are protective in vivo. Sheahan's group recently reported that, in a mouse model, immune effector function does, for some antibodies, help protect against the virus. "There is a difference in efficacy, yes," says Davide Robbiani, director of the Institute for Research in Biomedicine in Bellinzona, Switzerland, and a coauthor on the paper. But "regarding efficacy and safety of antibody effector functions, it's early to be conclusive."

Besides effector function, another big divide in the field is cocktails. In addition to Regeneron, Lilly is also testing an antibody cocktail, as is AstraZeneca, which is developing two antibodies discovered at Vanderbilt University. An antibody pair can bind the virus spike protein at two distinct epitopes to overcome drug resistance if one mutates. "The virus may evolve; the virus may find ways to escape the treatment with monoclonal antibodies, especially if a single monoclonal antibody is being used," says Robbiani.

But Vir is testing single-antibody therapy, in part because its antibody binds to a viral epitope that rarely mutates, says Scangos. It will be "more difficult for the virus to escape this antibody than a cocktail of two antibodies that Regeneron or Lilly or others are bringing forward," he says. "Two isn't better than one, necessarily. Quality matters."

Centivax is also going with a single antibody. Glanville points out that resistance mutations to the anti-RSV mAb, Synagis, are rare. For SARS-CoV-2, as based on an analysis of viral sequences in public databases, "I think the amount of escape variants is going to be pretty low, and in the low cases that happens, there are these other antibodies people can take," says Glanville. "My goal is to make a medicine that's less expensive, [so] more people can take it."

Furthermore, at lower doses, intravenous infusions could be replaced by intramuscular (IM) or subcutaneous injections. Regeneron is also teaming up with gene therapy pioneer

\section{AstraZeneca joins Russia to boost coronavirus vaccine}

AstraZeneca will begin clinical trials in December on a combination regimen that includes their own COVID-19 vaccine candidate and part of Russia's Sputnik V. The UK-based pharma announced it would explore combinations of different types of vaccines following phase 3 data published in The Lancet showing its own vaccine yielded lower efficacy than that achieved by mRNA vaccines from Moderna and Pfizer/BioNTech. The US Food and Drug Administration granted Emergency Use Authorization for the Pfizer/BioNTech vaccine on 11 December and for the Moderna vaccine on 18 December.

Russia’s Sputnik V vaccine was developed by the Gamaleya Research Institute and backed by the sovereign Russian Direct Investment Fund. It received a controversial approval from the Russian government in August before phase 3 efficacy trials had begun. The vaccine, which follows a prime-and-boost approach, combines two slightly different human adenovirus-based vectors: a recombinant adenovirus serotype 26 (Ad26) and serotype 5 (Ad5). Each bears genetic fragments that encode the SARS-CoV-2 spike glycoprotein. Interim results announced on 14 December show Sputnik V with an efficacy of $91.4 \%$ in phase 3 trials, but data have not yet been published.

Also in December, AstraZeneca and University of Oxford reported preliminary data from a phase 3 trial of their vaccine candidate, AZD1222. The vaccine showed $70.4 \%$ efficacy overall after two doses. But the company also revealed a puzzling finding: a subpopulation receiving a lower first dose, due to an error in dosing, experienced $90 \%$ efficacy.

AZD1222 uses the chimpanzee ChAdOx1 adenovirus vector to express the spike protein. AstraZeneca will test its own vaccine with Gamaleya's Ad26 vector. Some experts have warned that the other vector used by Gamaleya, Ad5, is derived from a human cold virus, which means some people may have a pre-existing immunity that could hamper response to the COVID-19 spike protein.

Published online: 11 January 2021 https://doi.org/10.1038/s41587-020-00804-4 